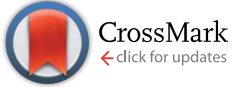

Cite this: RSC Adv., 2015, 5, 1462

Received 5th November 2014

Accepted 27th November 2014

DOI: $10.1039 / c 4 r a 13936 d$

www.rsc.org/advances

\section{Structural hysteresis in dragline spider silks induced by supercontraction: an X-ray fiber micro- diffraction study}

\begin{abstract}
Sujatha Sampath ${ }^{\star a b}$ and Jeffery. L. Yarger ${ }^{b}$
Interaction with water causes shrinkage and significant changes in the structure of spider dragline silks, which has been referred to as supercontraction in the literature. Preferred orientation or alignment of protein chains with respect to the fiber axis is extensively changed during this supercontraction process. Synchrotron X-ray micro-fiber diffraction experiments have been performed on Nephila clavipes and Argiope aurantia major and minor ampullate dragline spider fibers in the native dry, contracted (by immersion in water) and restretched (from contracted) states. Changes in the orientation of $\beta$-sheet nanocrystallites and the oriented component of the amorphous network have been determined from wide-angle X-ray diffraction patterns. While both the crystalline and amorphous components lose preferred orientation on wetting with water, the nano-crystallites regain their orientation on wetrestretching, whereas the oriented amorphous components only partially regain their orientation. Dragline major ampullate silks in both the species contract more than their minor ampullate silks.
\end{abstract}

\section{Introduction}

Supercontraction is the phenomenon in which unrestrained spider dragline silk contracts to about $50 \%$ of its original length when wetted with water or exposed to highly humid environments. ${ }^{1-4}$ In restrained conditions, exposure to water or high humidity causes substantial mechanical stress in the silk fibers. $^{3,5}$ The biological function of supercontraction is still under debate, proposed functions being, taking up the slack in the webs exposed to moisture, restoring tension and shape of the webs after prey capture, and counter-balancing extensional forces created by moisture on the webs. ${ }^{6,7}$ With a unique combination of high elasticity, mechanical strength and toughness, spider silks are remarkable biomaterials with extraordinary mechanical properties. ${ }^{8-10}$ Tensile properties studies on major ampullate silks from different spider species and between silks from different glands of the same spider species are well documented. ${ }^{7}$

The superior mechanical properties of spider silks are attributed in large part due to its mesoscopic to nanoscale hierarchical structuring. ${ }^{\text {9-12 }}$ Supercontraction causes substantial change in the mechanical properties making it rubber-like, with decreasing elastic modulus, stiffness and increase in extensibility. ${ }^{\mathbf{1 3 , 1 4}}$ Water is hypothesised to plasticize the silk structure by breaking hydrogen bonds in the hydrophilic

${ }^{a}$ Dept. of Physics, University of Wisconsin-Milwaukee, Milwaukee, WI 53211, USA. E-mail:sampath@uwm.edu; sujatha.sampath@asu.edu

${ }^{b}$ Department of Chemistry and Biochemistry, Magnetic Resonance Research Center, Arizona State University, Tempe, AZ, 85287-1604, USA. E-mail: jyarger@gmail.com regions. ${ }^{15-18}$ Re-alignment of protein chains and loss of preferred orientation results from supercontraction. ${ }^{\mathbf{1 6 , 1 9 - 2 1}}$ The biological role and significance of supercontraction in the spinning and drawing of the fiber from the concentrated solution in aqueous environment in silk gland and its role in maintaining the integrity of the spider webs in rain and or moisture is under debate., ,3,7,22-28 Supercontraction causes a permanent change in the microstructure of the silk fiber compared to its dry native structure..$^{\mathbf{1 6 , 1 9 , 2 0 , 2 9 , 3 0}}$ Supercontraction has been suggested to be a ground state of the fiber through which the tensile properties the silk fiber can be reproduced in a predictable, repeatable manner irrespective of the loading history of the fiber. ${ }^{3,5,6,23}$ Controlled supercontraction has been suggested as a mechanism by which silk properties can be tailored and used effectively in bioinspired synthetic fibers. ${ }^{6,23}$

In order to understand what makes spider silks mechanically superior, it is imperative to understand its structural organization. Synchrotron X-ray fiber diffraction is a versatile technique to understand the nano-scale structuring of the spider silk fiber. Previous X-ray studies have shown the molecular organization of spider's dragline silk to consist of a semicrystalline structure in which nano-crystallites made up of $\beta$ pleated sheets are embedded in an amorphous matrix. ${ }^{31-35}$ Silk from the major ampullate gland (commonly called dragline silk) of orb-weaving spiders have been studied the most, due to the relative ease of availability and superior tensile properties of the silk. Knowledge of silk structure from other silk glands, which have different protein compositions provides an excellent opportunity to study the protein sequence-structure-properties correlation. This knowledge will help to synthesize biomimetic 
fibers with desired structure and function. We have previously carried out a detailed X-ray structural investigation between major ampullate (MA) and minor ampullate (MiA) silks in two different spider species Nephila clavipes and Argiope aurantia. ${ }^{35}$ Structural similarities and differences between MA and MiA silks within the same species and between silks from two different species have been discussed in detail. ${ }^{35}$

In this paper, we report the nano-structure of MA and MiA silks (both of which are used in web formation) of Nephila clavipes and Argiope aurantia in the supercontracted and wetrestretched states, and compare it to their native dry state structure. Previous X-ray diffraction studies have shown that when the supercontracted silk is re-wetted and stretched to its original length, the silk structure which changes upon supercontraction, reverts back to its original dry-state structure. ${ }^{19}$ Macroscopic tensile properties have been reported to be similar when silks have been wet-restretched from the supercontracted state. ${ }^{4-6,36,37}$ We have carried out careful measurements and analysis and found that the silk structure regains its native structure only partially, there are differences between the structure of the dry native silk structure and restretched silk structure as a consequence of the supercontraction process.

It is critical to understand the molecular structural rearrangement through supercontraction, to design synthetic fibers for specific functions, which either takes advantage of the shrinkage or avoids shrinkage for other applications.

\section{Experimental}

\section{Spider specimen and forced silking techniques}

Spiders of two different species - Nephila clavipes and Argiope aurantia were forcibly silked at a rate of $2 \mathrm{~cm} \mathrm{~s}^{-1}$ to collect silks from both the MA and MiA glands. Silking was performed under a dissection microscope to ensure that the MA and MiA silks were separated during the entire process. Spiders were silked every other day on average and were fed a cricket during silking. It is to be noted that spiders produce MiA silks in much lesser quantity than the MA silks.

\section{Wide angle X-ray diffraction (WAXD)}

X-ray fiber diffraction was performed on Nephila clavipes and Argiope aurantia MA and MiA silks in three different states of the silk fiber: The dry native silk, supercontracted silk and the wetrestretched silk (stretched in water from the supercontracted state), at the BioCARS 14BM-C beamline in the Advanced Photon Source at Argonne National Laboratory, Argonne IL, USA. The wavelength of the X-ray beam was $0.9 \AA$ with a flux of $6 \times 10^{11}$ photons per sec and the beam size on the sample was $150 \times 200 \mu \mathrm{m}$. Data were recorded using an ADSC Quantum-315 detector. A bundle of 20 individual fibers were held taut (but unstretched) in a metal frame made out of a thick wire and mounted on the sample stage with the fiber axis normal to the $\mathrm{X}$-ray beam. The sample to detector distance was $200 \mathrm{~mm}$. Data collection time was 60 seconds for one image for the MA silks and 60 seconds for MiA silks. Background measurement was performed with the sample moved out of the incident X-ray beam and the image was recorded under the same conditions as used with the sample in the beam. Multiple images were taken to get better statistics and improve on the signal/ background ratio. $\mathrm{CeO}_{2}$ powder was used for instrument calibration. The 2D WAXD patterns were analysed using the software package FIT2D. ${ }^{38}$ Azimuthal 1D profiles were obtained from the deconvolution of 2D WAXD images using FIT2D. 1D Azimuthal profiles are integrated intensity as a function of all azimuthal angles over a very thin annular ring centered at the peak maximum of the desired reflection. ${ }^{31,32,35}$ Microcal Origin was used for the deconvolution of the 1D X-ray data and peak fitting. The peaks were fitted with Gaussian functions using nonlinear least squares fitting. ${ }^{35}$

Before wetting with water, the length of the metal frame which held the fiber bundle was reduced such that the silk which was previously held taut was now hanging loosely. Distilled water was added to the silk by a pipette and the silk contracted freely and reduced in length. After the contraction of the silk fibers, any excess water was removed with a paper towel and the silk was let to dry at room temperature for ten minutes. WAXD patterns were taken under the same conditions described above for the native silk. To restretch the silk, the supercontracted silk was wetted with distilled water with a pipette, and cautiously stretched to as close as was possible to its original length (0.98 of original length) and dried as described for the supercontracted silk, before performing WAXD.

Extent of supercontraction is defined in this paper (in accordance with previous works $\left.{ }^{19,28}\right)$ as $\left(L_{0}-L_{\mathrm{c}}\right) / L_{0}$, where $L_{0}$ is the original dry native state length of the fiber and $L_{\mathrm{c}}$ the length of the supercontracted/contracted unrestrained fiber.

\section{Results and discussion}

\section{Nephila clavipes: MA and MiA silks}

Fig. 1a-c show 2D WAXD patterns of Nephila clavipes MA silk in the dry (native), supercontracted and restretched (from supercontracted) states, respectively. The diffraction pattern of the native dry silk depicts a semi-crystalline morphology characterized by Bragg reflections and an amorphous halo, as reported in our study and in other studies previously. ${ }^{31-35}$ According to the prevalent structural model, spider silk has a hierarchical structure wherein each silk fiber is made up of many fibrils coaxially parallel to the fiber axis and each fibril composed of well-aligned nano-crystalline domains connected by an amorphous matrix, comprised of both an oriented and an isotropic component. ${ }^{39}$ Intense reflections are observed along the equator (perpendicular to the fiber axis). Along the meridian (parallel to the fiber axis) the main (002) reflection is strong, while subsequent higher order reflections are weaker. Crystalline reflections are broad due to the small size of the crystallites. ${ }^{31,35}$ Reflections comprising the crystalline fraction correspond to $\beta$-poly(L-alanine) structure and were indexed based on an orthogonal unit cell with the $c$-axis as the fiber axis. Crystallite size, orientation distribution of the protein chains about the fiber axis (in both crystalline and oriented amorphous components), and percentage crystallinity in the silk fiber can be determined from WAXD. ${ }^{31,32,35}$ 

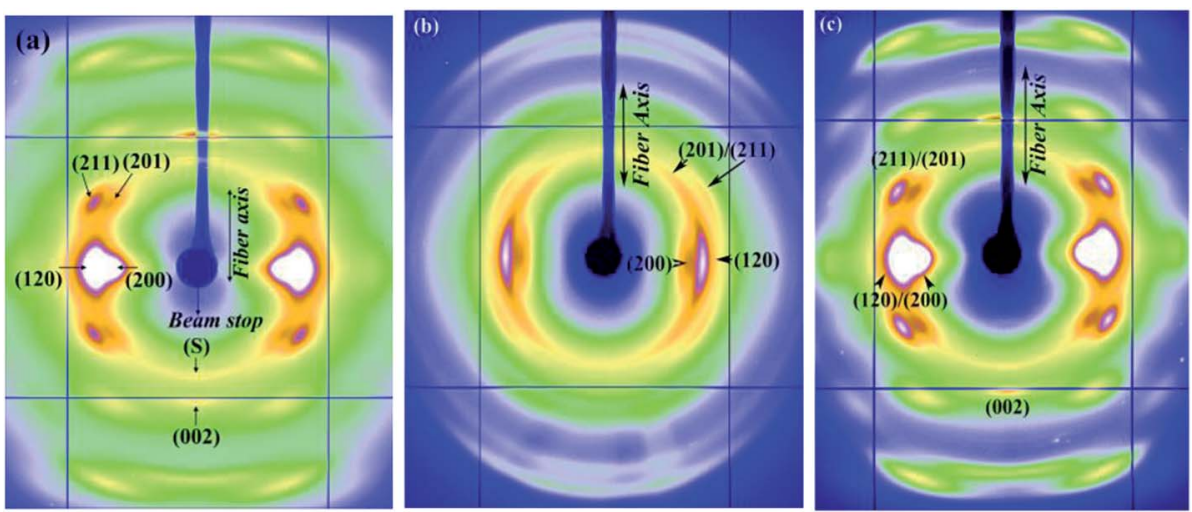

Fig. 1 (a-c) WAXD patterns of Nephila clavipes MA silk (a) dry native silk (b) supercontracted silk (c) wet-restretched silk.

On wetting with water in the unrestrained state, $\left(L_{0}-L_{\mathrm{c}}\right) / L_{0}$ for the MA silk was 0.46. In the supercontracted state (Fig. 1b), crystalline reflections occur at the same $d$-spacings as in the native dry silk. ${ }^{35}$ Substantial azimuthal broadening of crystalline reflections are observed in the supercontracted fiber (Fig. 1b), indicating reduction or loss of preferred orientational order of the protein chains with respect to the fiber axis, compared to the dry native silk. ${ }^{19,29}$ In the restretched state (Fig. 1c), the WAXD pattern resembles that of the native dry silk, crystalline reflections occur at the same $d$-spacings and have the orientational order of that of the dry silk. To determine if the dry silk and the restretched silk are identical at a molecular level, the amorphous matrix has to be taken in consideration in addition to the crystalline component. Changes in the amorphous component between the dry and restretched states are not as evident visually from the $2 \mathrm{D}$ WAXD pattern (unlike crystalline reflections) because of the diffuse nature of this diffraction. Careful data analysis is required to observe changes in the non-crystalline diffraction intensities, and this is discussed in the following sections.

Fig. 2a and b show the 2D WAXD patterns of Nephila clavipes MiA silk in the dry and contracted states. For the Nephila clavipes MiA silk $\left(L_{0}-L_{\mathrm{c}}\right) / L_{0}$ was 0.16 . Crystalline reflections present in the dry silk are present in the contracted state and at the same $d$-spacings, ${ }^{32}$ however they are broadened due to loss of orientation during wetting of the silk. In the case of MiA silk, the term contracted is used, to follow the convention used in the silk research field where supercontraction is used when the fiber contracts to $30 \%$ or higher of their original length on wetting. On wetting with water, both MA and MiA silks show loss of orientational order of the protein chains w.r.t. to the fiber axis. Positions of the crystalline reflections and crystallinity are not affected because water is not able penetrate the nano-crystallites. Supercontraction is known to cause a loss of orientation of the protein chains in the oriented amorphous region as evidenced by different characterization techniques (XRD, NMR, FTIR). ${ }^{2,16,19,20,30}$

Orientation or alignment of protein chains in the fiber is an important factor in determining the mechanical properties of spider silk. ${ }^{2,40}$ While chemical composition, spinning and post spin drawing conditions together determine the physical properties of spider silk fiber, for a given composition it is known that faster spinning speeds facilitated better orientation of the protein chains., ${ }^{2,31,41}$ Fibers having better preferred molecular chain orientation ${ }^{42,43}$ are known to have better mechanical, ${ }^{44}$ electrical ${ }^{45}$ and thermal properties. ${ }^{46}$ Molecularchain orientation is therefore a crucial parameter in influencing the physical properties of the spider silk fibers. Supercontraction caused by water is known to cause loss of orientation and change mechanical properties substantially. ${ }^{2}$ Liu et al. ${ }^{2}$ have correlated the fiber's 'maximum capacity to shrink in unrestrained conditions' to orientational order of the molecular chains in the fiber. The effect of orientation is also seen in synthetic fibers (e.g., Nylon, PET), where thermal shrinkage caused by heat during processing is associated with loss of orientation of molecular chains within the amorphous network in the fiber. ${ }^{47-49}$ It is therefore imperative to understand and characterize orientational order through the supercontraction process. WAXD is an excellent technique to determine the orientational order (orientation between the polypeptide backbone of the protein chains and the fiber axis) in both the crystalline and oriented amorphous components. ${ }^{19,31-35}$

\section{Orientational order}

Dry native MA silk. Orientation of the protein chains about the fiber axis can be determined from azimuthal broadening (FWHM) of the (200)/(120) equatorial reflections (in both, the
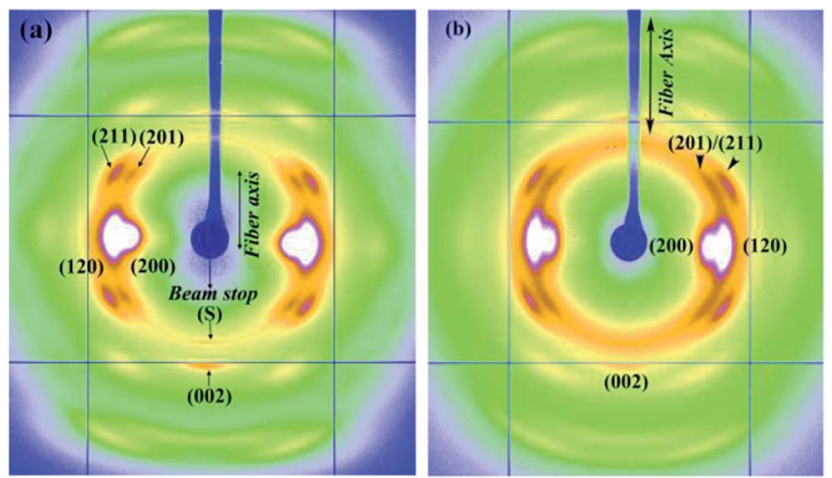

Fig. 2 (a and b) WAXD patterns of Nephila clavipes MiA silk (a) dry native silk (b) contracted silk. 
nano-crystallites \& the oriented amorphous component). In the case of the nano-crystallites, orientational order can be expressed quantitatively in terms of Herman's orientation function $f_{\mathrm{c}}=\left(3\left\langle\cos ^{2} \varphi\right\rangle-1\right) / 2$, where $\varphi$ is the angle between the $c$ axis and the fiber axis. ${ }^{42}$ The parameter $f_{\mathrm{c}}$ is 0 for no preferred orientation in fibers and 1 if all crystallites are perfectly aligned with respect to each other. $\left\langle\cos ^{2} \varphi\right\rangle$ can be obtained from azimuthal width of the two strongest equatorial reflections ${ }^{31,32,44}$ (200)/(120) based on the equation $\left\langle\cos ^{2} \varphi\right\rangle=1-A\left\langle\cos ^{2} \varphi_{1}\right\rangle-B$ $\left\langle\cos ^{2} \varphi_{2}\right\rangle$, where $A=0.8, B=1.2 .^{31,32}$ Orientation function of the amorphous component has been calculated as done with dry native silks in ours and other previous studies. ${ }^{19,35}$

Fig. 3a and b show intensity as a function of angle measured from the equator at the radial position of the (120) and (200) peaks, respectively, for Nephila clavipes MA dry native silk. (Annular region used for integration are shown in the insets) Both (120) and (200) peaks have been fit with two Gaussian functions, one wider than the other, according to the structural model used in previous studies on spider silks. ${ }^{31,35}$ It has been shown by Grubb et al. ${ }^{31}$ that the narrower Gaussian dominates at the equator and corresponds to the crystalline material ( $I_{120}$ or $I_{200}$ ), and the broader Gaussian corresponds to the oriented amorphous material, which dominates about $20^{\circ}$ off the equator. In the case of the (120) peak profile, small subsidiary peaks due to residual intensity from the (201) reflections were treated as individual Gaussian functions. Values of orientation function for the crystallites and the oriented amorphous material are 0.98 and 0.808 respectively.

Supercontracted MA silk. Azimuthal scans at the positions of the equatorial reflections (120) and (200) for the supercontracted Nephila clavipes MA silk are shown in Fig. 3c and d, respectively. As seen from the $2 \mathrm{D}$ WAXD pattern in Fig. 1b, there is significant broadening of the crystalline reflections and considerable overlap between reflections. Deconvolution of individual peaks in Fig. $3 \mathrm{c}$ is challenging due to the overlap of the subsidiary (201) peaks with the main (120) peak. Ambiguity in the determination of FWHM due to overlapping peaks is resolved by fixing the position of the subsidiary peaks to the value of the well-oriented dry native fibers as a constraint in the fitting process. ${ }^{19}$ The fitting procedure and calculation of the orientation parameters have been performed in the similar manner as for the native dry silk (Fig. 3a and b). Values of orientation function for the crystallites and the oriented amorphous material 0.926 and 0.703 for the oriented amorphous material respectively, in the supercontracted Nephila clavipes MA silk.

Wet-restretched MA silk. Fig. 3e and f show the 1D azimuthal scans of the $(120) /(200)$ peaks for the Nephila clavipes MA silk which has been restretched to its original length by re-wetting in water. As seen in the 2D WAXD pattern, (Fig. 1c) crystalline reflections regain their orientation as in the dry native fiber, and from the 1D azimuthal profile (Fig. 3e), it is seen that the subsidiary 211 peaks are well separated from the (120) peak (overlapping in the supercontracted fiber). Orientation of the protein chains in the crystalline and amorphous fractions have been calculated as for the dry fiber. Values of orientation function for the crystallites and the oriented amorphous material are 0.978 and 0.738 respectively, in the restretched silk.

Dry and contracted MiA silk. Fig. $4(\mathrm{a}, \mathrm{b})$ and (c, d) show the $1 \mathrm{D}$ azimuthal profiles at the positions of the equatorial reflections (120) and (200) for the dry and contracted Nephila clavipes MiA silk respectively. MiA silk contracted to a lesser extent than the MA silk. From the 2D WAXD pattern of the contracted MiA silk (Fig. 2b) azimuthal broadening of the reflections are observed, but to a lesser extent than seen in the case of MA silk. Peak fitting and calculation of the orientation parameters have been done as for the MA silk. Values for orientation function for the dry native Nephila clavipes MiA silk are $f_{\mathrm{c}}=0.975$ and $f_{\mathrm{am}}=0.758$ for the crystallites and oriented amorphous material, respectively. For the contracted fiber, $f_{\mathrm{c}}$ is 0.957 and $f_{\mathrm{am}}$ is 0.519. In the dry native state, protein chains in the nanocrystallites and the oriented amorphous component are better oriented in the MA silk than in the MiA silk. However, on wetting, the loss of orientation is larger for the MA silk than for the MiA silk.

Structure of the wet-restretched MiA silk could not be studied because the fiber broke while re-stretching. Breaking of the contracted fiber while wet-restretching also occurred in the case of MiA silk from another species Argiope aurantia, studied in this paper, indicating MiA silks are not structurally resilient to wet-stretching as the MA silks.

Interaction with water has been hypothesized to have a plasticizing effect on the silk fiber by causing breaking or disruption of hydrogen bonds in the hydrophilic regions in the amorphous matrix. ${ }^{\mathbf{1 5 - 1 8}}$ The $\beta$-sheet nanocrystallites are hydrophobic and water impenetrable and hence retain their integrity as crystallites in the supercontracted state. Strong polar solvents like water are known to penetrate the amorphous network and break the hydrogen bonds within the network. ${ }^{50}$ Breaking of hydrogen bonds between protein chains in the amorphous matrix lowers the network connectivity due to loss of structural constraints imposed by hydrogen bonds in the matrix. This would in turn enhance mobility of the protein chains in the matrix, resulting in a floppy/flexible matrix with lower bonds holding the network as constraints. In this floppy matrix state, the hydrophobic nanocrystallites are connected by lower number of bonds within the network (lower network connectivity, rigidity) and hence have a higher degree of freedom in their orientation. In this condition of the fiber, the protein chains are not restricted to the preferred alignment parallel to the fiber axis. In the supercontracted state, loss of preferred orientation manifests as azimuthal broadening of the crystalline reflections in the WAXD patterns as seen in both Nephila clavipes MA and MiA silks. In MA silks however, on wetrestretching, the broken hydrogen bonds are thought to form again between the protein chains, constraining the protein chains to realign parallel or in the preferred direction w.r.t. the fiber axis, thus imposing rigidity in the matrix. As seen from Fig. 1c and $3(\mathrm{a}, \mathrm{b})$, the realignment of the nano-crystallites follows this trend in the MA silk, however, we found that the oriented amorphous component does not regain its dry-native state value. One implication of this finding is that the hydrogen bonds which were broken due to wetting the fiber 

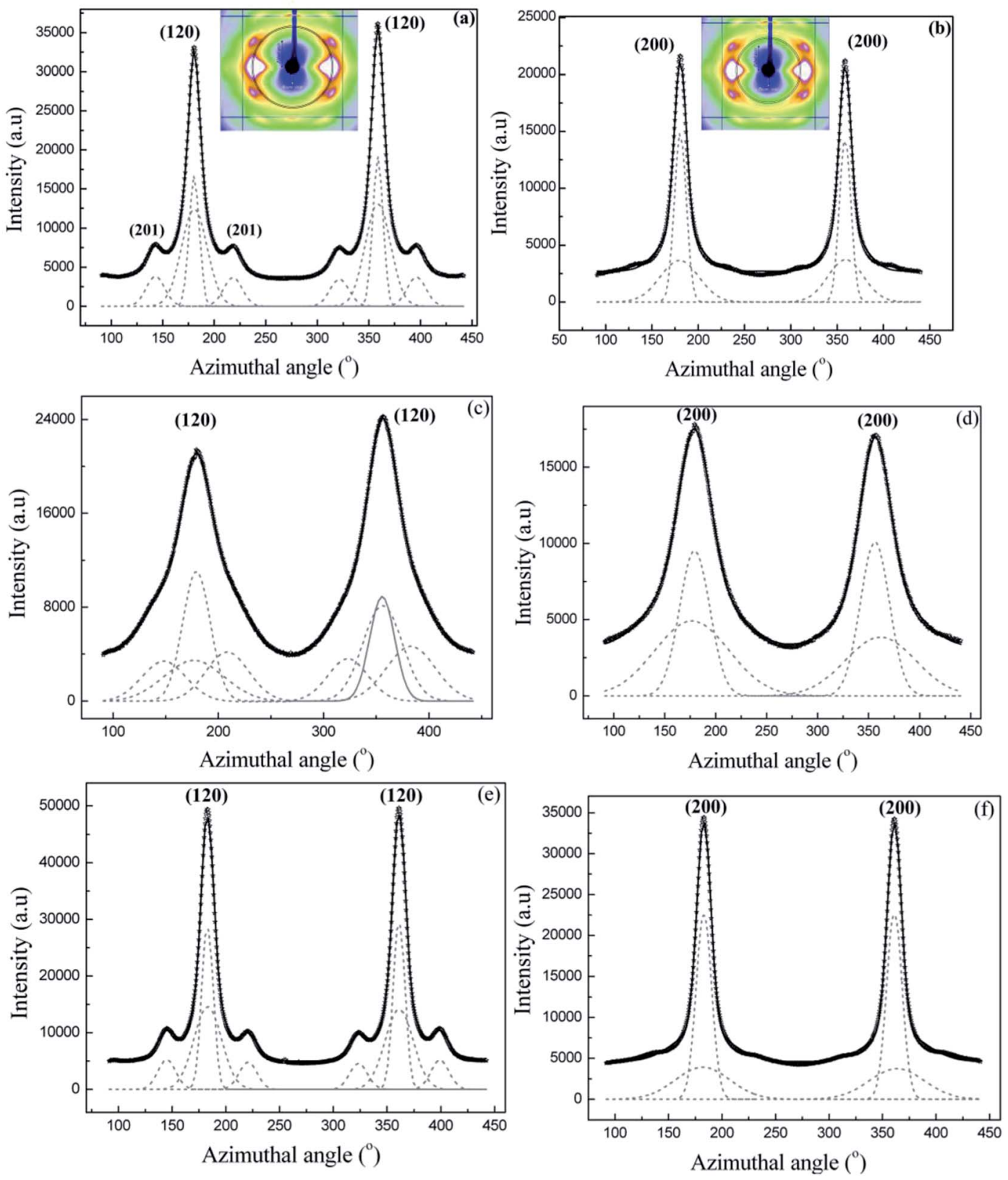

Fig. 3 (a-f) 1D azimuthal intensity profile of the radially integrated (120) and (200) peaks with Gaussian fits for Nephila clavipes MA silk. (a) (120) peak dry native silk. Inset shows the annular section chosen for the integration. (b) (200) peak, dry native silk (c) (120) peak in supercontracted silk (d) (200) peak in supercontracted silk (e) (120) peak in the wet-restretched silk (f) (200) in wet-restretched silk.

with water causing supercontraction, only some but not total percentage of them have re-formed when the fiber was restretched in water to its original length. Another possibility is that the fraction of the oriented amorphous component differs between the native dry silk and the fiber restretched after supercontraction.

A 'mechanical ground state' has been proposed for MA silk fiber by Guinea et $a{ }^{3}{ }^{3}$ 'This basic, or initial, state can be recovered regardless of the fiber loading history by simply subjecting the unrestrained fiber to water immersion and subsequent drying (Elices et al. .). ${ }^{5}$ It has also been found that the whole range of tensile properties exhibited by these fibers can be obtained in a reproducible way by simply stretching the fibers in water from the ground state'. ${ }^{6}$

Supercontraction in MA silks has been seen as a means to reach this 'ground state' of the fiber. ${ }^{3,5,6}$ It is expected that microstructure of the MA silk follow a similar trend when subjected to dry-wet-dry cycles. This would imply that the structure of the fiber would return to its original dry-native state structure, if stretched in water from its supercontracted state to its original length. However, our results show that while the nanocrystallites follow this trend, the oriented amorphous component does not regain its orientation to its dry state value. ${ }^{2}$ 

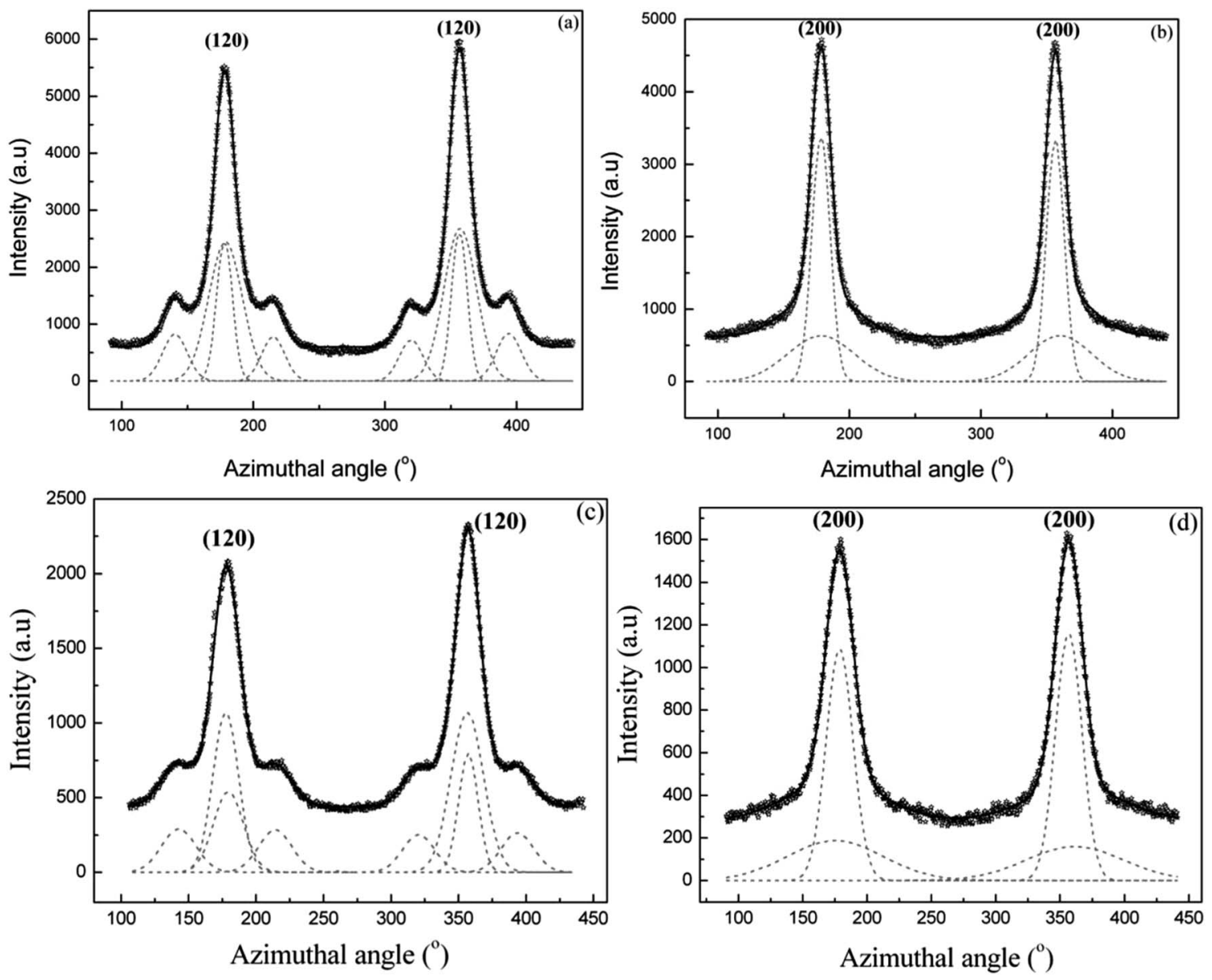

Fig. 4 (a-d) 1D azimuthal intensity profile of the radially integrated (120) and (200) peaks with Gaussian fits for Nephila clavipes MiA silk. (a) (120) peak dry native silk (b) (200) peak dry native silk (c) (120) peak in contracted silk (d) (200) peak in contracted silk.

This finding has profound practical implications in biomemtic efforts to produce synthetic fibers based on natural silk sequences. Identifying specific sequences in the amorphous matrix causing this hysteresis in protein chain orientation will help with design of biomimetic fibers to circumvent this behaviour. The amorphous matrix, which is significant fraction of the silk $(\sim 70 \%)$, plays an important role in the fiber's mechanical properties. ${ }^{2}$

\section{Comparison between Nephila clavipes MA and MiA silks}

MA and MiA silks are each composed of two proteins, major ampullate spidroin 1 and 2 (MaSp1/MaSp2) and minor ampullate spidroin 1 and 2 (MiSp1/MiSp2), respectively. MaSp1 and MaSp2 contain poly-alanine (poly-A) runs up to 10 residues long and glycine-glycine-X (GGX) runs (where $\mathrm{X}$ ) glutamine $(\mathrm{Q})$, leucine $(\mathrm{L})$, or tyrosine $(\mathrm{Y})$, with MaSp2 also having glycineproline-glycine-X-X (GPGXX) motifs (where XX) (G-Y or $\mathrm{Q}-\mathrm{Q}){ }^{51-53}$ MiSp1 and 2 are made up of (i) poly-A repeats that are shorter than MA silk, (ii) GGX motifs (where X) A, Q, or Y), (iii) long poly(glycine-alanine) (poly-(GA) regions similar in length to the poly-A repeats in MA silk, and (iv) a Ser-rich nonrepetitive spacer region ("spacer"). The poly-A regions in MA and MiA silk have been shown to be in a $\beta$-sheet structure by solid-state
NMR. ${ }^{16,30}$ Solid-state ${ }^{2} \mathrm{H}$ NMR also points to highly ordered $\beta$-sheet, as well as disordered Ala rich regions in MA silk. ${ }^{16,54,55}$

While both MA and MiA silks have a semi-crystalline morphology, differences in crystallinity and orientation of protein chains have been found from our previous study. ${ }^{32}$

(i) Nephila clavipes MiA silk is more crystalline than the Nephila clavipes MA silk and the $\beta$-sheet nanocrystallites are bigger in MiA silk than in MA silk.

(ii) While the orientation of the $\beta$-sheet nano-crystallites is comparable between MA and MiA silks, the oriented amorphous component is found to be better oriented in the MA silk (Table 1).

Mechanical properties measurements have indicated that MA silk is stronger with higher ultimate strength, but is less compliant than MiA silk, which has a higher extensibility and higher Young's modulus, but lower strength. ${ }^{56,57}$ In terms of its protein sequence, poly-alanine (poly-Ala) regions have higher binding energy than poly-Glycine-Alanine (poly-Gly-Ala) regions. ${ }^{58} \mathrm{MA}$ silk has more poly-Ala in their sequence, which may account in part for better orientation of protein chains due to better inter-chain bonding and consequently the higher ultimate strength of MA silk.

While there are subtle differences in the mechanical properties of MA and MiA silks tested in air, their mechanical 
Table 1 Herman's orientation function and crystallinity parameters for Nephila clavipes and Argiope aurantia MA and MiA silks

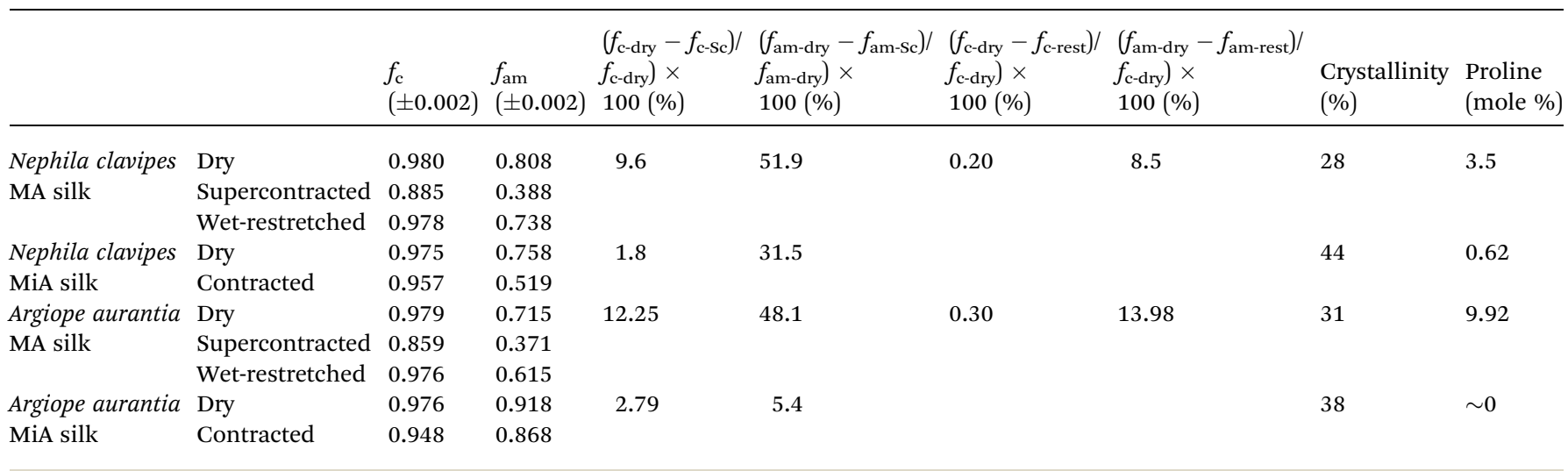

properties are significantly different when exposed to water. $^{25,42-44}$ Tensile properties of MA silks are affected significantly more than the MiA silks, due to the plasticizing effect of water during supercontraction, with a significant reduction in the initial elastic modulus in MA silks. ${ }^{15,26,28}$

Guinea et al. have recently studied the recovery and reversibility of deformation of MA and MiA silks during loadingunloading-reloading cycles. ${ }^{25}$ MA silks were found to recover their tensile properties when stretched in water through loading-unloading-loading cycles, whereas stretching in water causes irreversible deformations in the MiA silks. ${ }^{25}$

Breaking of MiA silks of Nephila clavipes and Argiope aurantia while re-stretching from contracted state in our study is commensurate with Guinea et al.'s study showing irreversible deformation caused by water on MiA silks of Nephila inaurata and Argiope trifasciata. ${ }^{25}$

From a structural perspective, higher crystallinity in Nephila clavipes MiA silk could in part be causing lower loss of orientation in when exposed to water. As the crystallinity increases, the network connectivity increases (the nano-crystallites forming the connectivity nodes), hence the structure can be thought of as a 'rigid' matrix with lower degrees of freedom. Lower crystallinity and consequently higher amorphous content in Nephila clavipes MA silk makes available higher number of hydrogen bonds in the hydrophilic amorphous network to be plasticized by water, resulting in breaking the network connectivity, accompanied by higher mobility and loss of orientation of the protein chains in the MA silk.

\section{Argiope aurantia: MA and MiA silks}

In an effort to study effects of different protein compositions on the silk structure, we chose to study MA and MiA silks from Argiope aurantia which has very different ratios of the proteins MaSp1/MaSp2 and MiSp1/MiSp2 than found in Nephila clavipes. ${ }^{59,60}$ Argiope aurantia and Nephila clavipes silks not only differ in the ratios of the two spidroins, but also differ in the amino acid proline (found only in the MaSp2), which in Argiope aurantia is known to be significantly higher than that found in Nephila clavipes. ${ }^{\mathbf{5 9 , 6 0}}$ The role of proline in the supercontraction phenomenon has been studied by various authors. ${ }^{7,59,60,62-65}$ Combination of the chemical sequence and processing conditions are thought to affect the resultant physical properties of spider silks, however for silks with different proline contents, the initial modulus decreased and capacity to shrink increased with increase in proline amounts in the silk. ${ }^{2}$ As proline forms a ring structure, with its side group attaching back to the polypeptide backbone, it introduces a steric constraint, thus restraining dense packing. When wetted with water, this stearic constraint imposed by the GPGXX motifs in MaSp2 are thought to allow for easier access to water molecules to penetrate the amorphous network and break the hydrogen bonds. The molecular structure of the GPGXX regions and the high mobility of these regions when exposed to water have been well studied using NMR spectroscopy. ${ }^{\mathbf{6 1}}$ The GPGXX regions are found in type IIA beta-turn structures in natural dragline silk. However, these regions become highly mobile when exposed to water.

Fig. 5a-c show the 2D WAXD patterns of Argiope aurantia MA silk in the dry (native), supercontracted and restretched states respectively. The native dry Argiope aurantia MA silk diffraction pattern (Fig. 5a) depicts a semi-crystalline structure with crystalline Bragg reflections and an amorphous halo (comprising of oriented and isotropic components) as in the case of the Nephila clavipes MA silk. ${ }^{35}$ On wetting with water in the unrestrained state, $\left(L_{0}-L_{\mathrm{c}}\right) / L_{0}$ for Argiope aurantia MA silk was 0.53 . In the supercontracted state (Fig. 5b), crystalline reflections occur at the same $d$-spacings as in the native dry silk. ${ }^{35}$ Similar to the trend seen in the Nephila clavipes silks, crystalline reflections in Argiope aurantia MA silk in the supercontracted state lose their preferred orientation with respect to the fiber axis, as seen from the azimuthal broadening of the reflections (Fig. 5b). WAXD pattern of the re-stretched Argiope aurantia MA silk (Fig. 5c), resembles that of the native dry silk (Fig. 5a). In the wetrestretched silk, crystalline reflections occur at same $d$-spacings and orientation of nano-crystallites is similar to that of native dry silk, however orientation of the 'oriented' amorphous fraction differs from that of the native dry silk.

Fig. $6 \mathrm{a}$ and $\mathrm{b}$ show the 2D WAXD patterns of Argiope aurantia MiA silk in the dry and contracted states. On wetting with water in the unrestrained state, $\left(L_{0}-L_{\mathrm{c}}\right) / L_{0}$ for Argiope aurantia MiA silk was 0.15. Argiope aurantia MiA silk as in the case of Nephila 

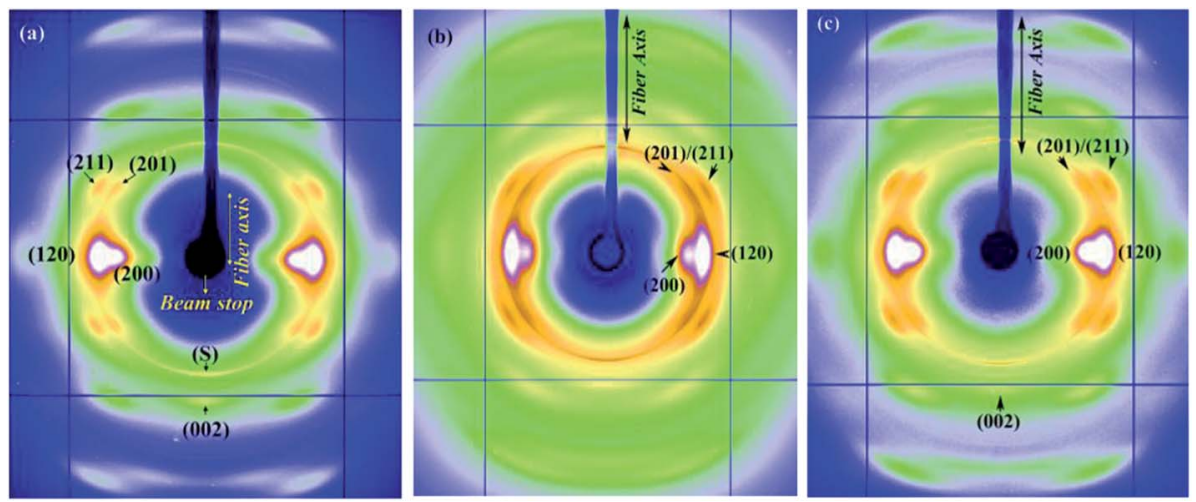

Fig. 5 (a-c) WAXD patterns of Argiope aurantia MA silk (a) dry native silk (b) supercontracted silk (c) wet-restretched silk.

clavipes MiA silk, contracted far less than that observed for the corresponding MA silk.

\section{Orientational order}

Argiope aurantia MA dry native silk. Fig. 7a and b show the 1D azimuthal intensity profile, as a function of angle measured from the equator at the radial position of (120) and (200) peaks respectively, for Argiope aurantia MA dry native silk. Overlap from subsidiary (201) peaks is lower than observed in Nephila clavipes MA silk (Fig. 3a). Peak fitting has been done in the same procedure as used for Nephila clavipes silks. Degree of orientation of both the crystalline and the oriented amorphous material have been determined from FWHM of the (120) and (200) peaks. Argiope aurantia MiA silk is more crystalline and less oriented w.r.t. the fiber axis as in the case of Nephila clavipes silks. ${ }^{32}$ Values of orientation function for the crystallites is 0.979 and 0.715 for the oriented amorphous material in the dry native Argiope aurantia MA silk.

Argiope aurantia MA supercontracted silk. Azimuthal intensity profiles at the positions of the equatorial reflections (120) and (200) for the supercontracted Argiope aurantia MA silk are shown in Fig. 7c and d, respectively. Crystalline reflections are broadened and there is overlap between reflections as also observed in the 2D WAXD pattern from Fig. 5b. Deconvolution
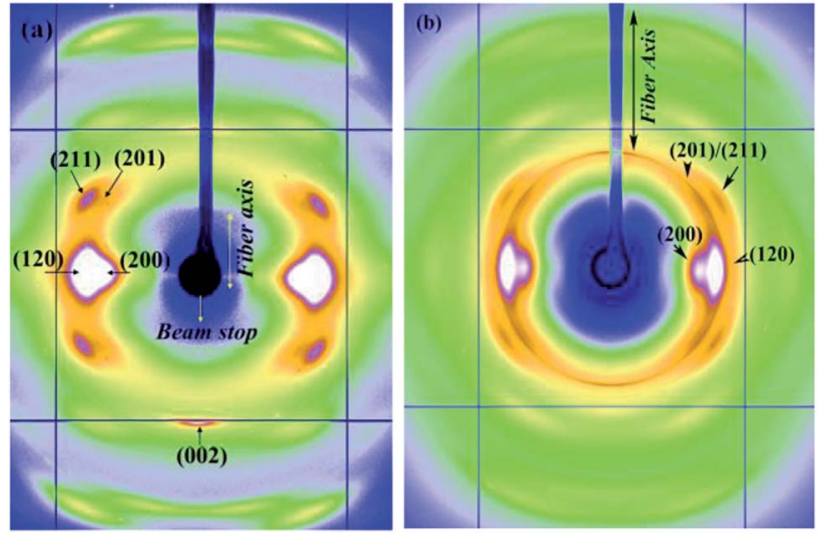

Fig. 6 (a and b) WAXD patterns of Argiope aurantia MiA silk (a) dry native silk (b) contracted silk. of individual peaks in Fig. 7c is challenging due to the overlap of the subsidiary (201) peaks with the main (120) peak. The fitting procedure and calculation of the orientation parameters have been performed as done for the Nephila clavipes supercontracted silk (Fig. 3c and d). Values of orientation function for the crystallites is 0.851 and 0.371 for the oriented amorphous material in the supercontracted Argiope aurantia MA silk.

Argiope aurantia MA restretched silk. Fig. 7e and f show the 1D azimuthal intensity profiles of $(120) /(200)$ peaks for the Argiope aurantia MA silk restretched to its original length (by re-immersion in water). Crystalline reflections regain their orientation as in the dry native fiber (also seen from 2D WAXDFig. $5 \mathrm{a}$ and 5c). Subsidiary 211 peaks in the restretched state are well separated from the (120) peak (overlap seen in the supercontracted state). Orientation of the protein chains in the crystalline and amorphous fractions have been calculated as for the dry fiber and the values listed in Table 1 . Values of orientation function for the crystallites and the oriented amorphous material are 0.976 and 0.615 respectively.

Dry native Argiope aurantia MiA silk. Fig. 8a and b show the azimuthal scans for the Argiope aurantia MiA silk in the dry state. The 2D WAXD patterns show semi-crystalline morphology similar to that of the Argiope aurantia MA silk and Nephila clavipes MA and MiA silks. Crystalline reflections are indexed to an orthorhombic unit cell. ${ }^{35}$ Values of orientation function for the crystallites and the oriented amorphous material are 0.976 and 0.918 in Argiope aurantia MiA dry silk. ${ }^{35}$

Contracted Argiope aurantia MiA silk. The 1D azimuthal scan of the equatorial (120)/(200) for the Argiope aurantia MA silk are shown in Fig. $8 \mathrm{c}$ and d. Peak fitting and the deconvolution of overlapped peaks have been done as for Nephila clavipes MA silk (described earlier). MiA silk contracts to a lesser extent than the MA silk in Argiope aurantia, similar to the trend seen between Nephila clavipes MA and MiA silks.

Values of orientation function for the crystallites and the oriented amorphous material are 0.948 and 0.868 respectively in Argiope aurantia MiA contracted silk.

In the case of the MiA the silk broke while trying to restretch in wet condition, a behaviour similar to that exhibited by Nephila clavipes MiA silk. 

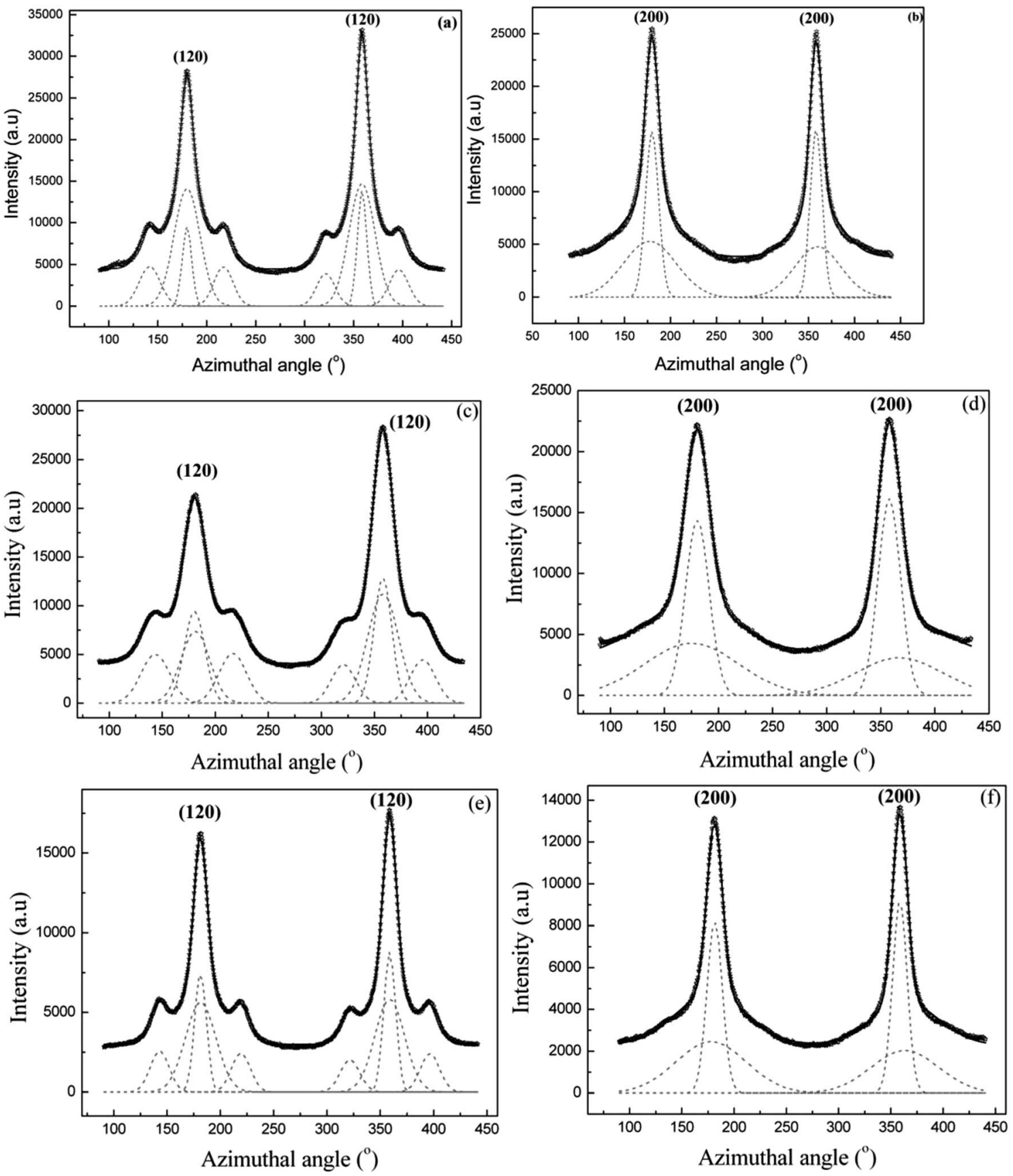

Fig. 7 (a-f) 1D azimuthal intensity profile of the radially integrated (120) and (200) peaks with Gaussian fits for Argiope aurantia MA silk. (a) (120) peak dry native silk. (b) (200) peak, dry native silk (c) (120) peak in supercontracted silk (d) (200) peak in supercontracted silk (e) (120) peak in the wet-restretched silk (f) (200) in wet-restretched silk.

\section{Comparison between Nephila clavipes and Argiope aurantia silks}

Table 1 shows the degree of orientation of the nano crystalline and oriented amorphous components in the major and minor ampullate silks of Nephila clavipes and Argiope aurantia in the dry, supercontracted and re-stretched states. To compare in quantitative terms on how supercontraction and wet-stretching affects the orientation in the four silks studied in this work, we have introduced the following quantities. Percentage change in the degree of orientation of the $\beta$-sheet nano-crystallites for each silk between the dry and supercontracted states is defined as, orientation function dry minus orientation function supercontrated $\left.\left[\left(f_{\text {c-dry }}-f_{\text {c-Sc }}\right) / f_{\text {c-dry }}\right) \times 100\right]$, and between the dry and re-stretched states as $\left.\left[\left(f_{\text {c-dry }}-f_{\text {c-rest }}\right) / f_{\text {c-dry }}\right) \times 100\right]$. Similarly for the oriented amorphous component, change in orientation function between the dry and supercontracted and dry and restretched states are defined as $\left.\left[\left(f_{\text {am-dry }}-f_{\text {am-sc }}\right) / f_{\text {am-dry }}\right) \times 100\right]$ and $\left.\left[\left(f_{\text {am-dry }}-f_{\text {am-rest }}\right) / f_{\text {c-dry }}\right) \times 100\right]$ respectively and shown in Table 1. 

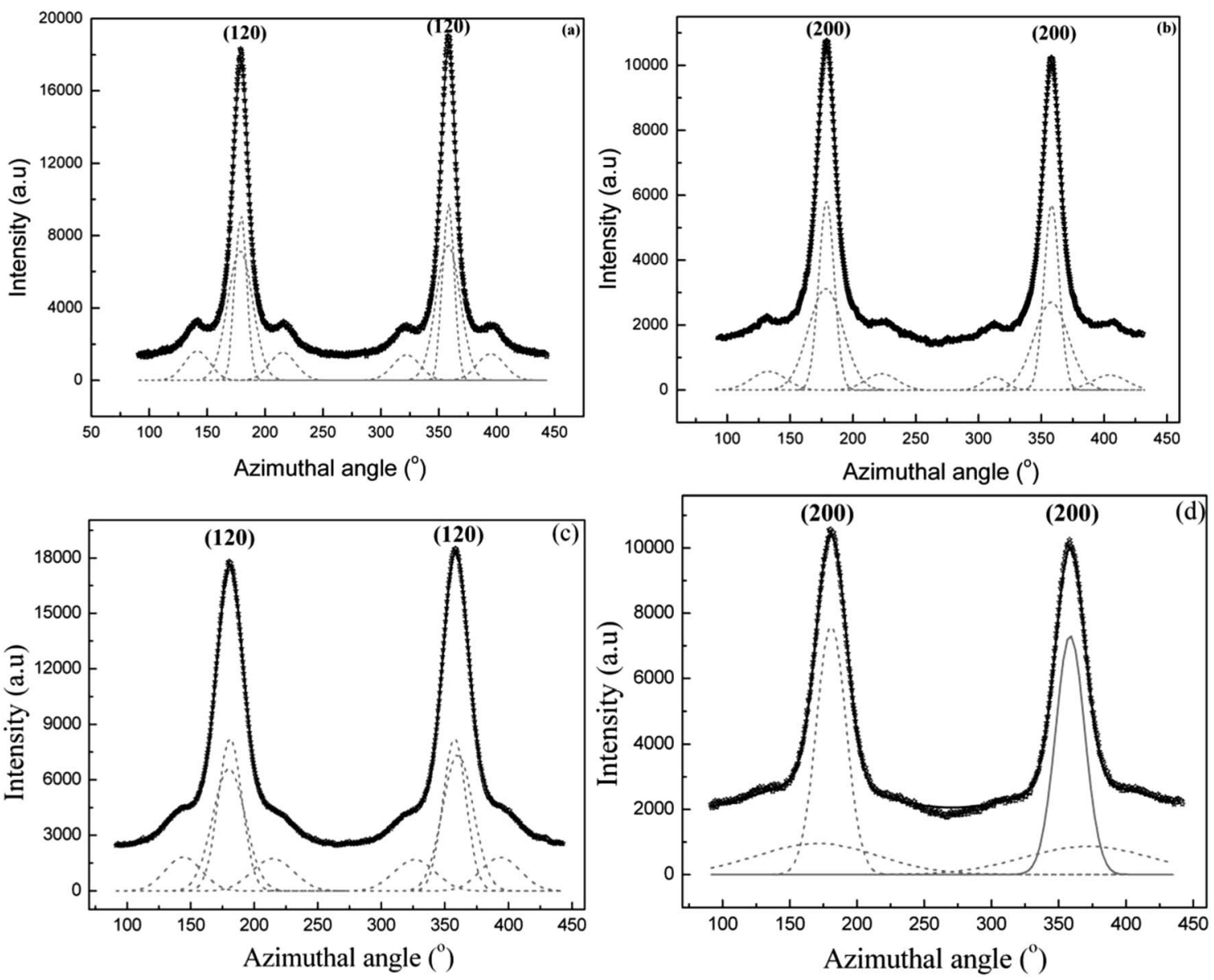

Fig. 8 (a-d) 1D azimuthal intensity profile of the radially integrated (120) and (200) peaks with Gaussian fits for Argiope aurantia MiA silk. (a) (120) peak dry native silk (b) (200) peak dry native silk (c) (120) peak in contracted silk (d) (200) peak in contracted silk.

Loss of orientation due to supercontraction is greater in the oriented amorphous component in all the four silks. Also MA silks of both Nephila clavipes and Argiope aurantia have a higher loss of orientation in the $\beta$-sheet nano-crystallites as well as the oriented amorphous component than their MiA silks.

Orientation change (loss) in the nano-crystallites due to water penetration is higher in the Argiope aurantia MA and MiA silks than their Nephila clavipes counterparts. This trend is reversed for the oriented amorphous component, Nephila clavipes MA and MiA silks have a higher loss of orientation of the amorphous component than Argiope aurantia MA and MiA silks (Table 1).

Comparing the orientation of protein chains in the crystallites and amorhous components between the re-stretched and the pre-supercontracted fibers in the MA silks, the $\beta$-sheet nanocrystallites regain the orientation to very close to its original state (difference being $0.2 \%$ and $0.3 \%$ for Nephila clavipes and Argiope aurantia MA silks respectively). However, there is a larger difference in the orientation of protein chains in the amorphous component, $8.5 \%$ (Nephila clavipes MA silk) and $14 \%$ (Argiope aurantia MA silk) lower than their native dry state values. This result indicates a structural hysteresis between the pre-supercontracted and the wet-restretched fiber. Nano- structure of the fiber does not recover its original presupercontraction structure when subjected to dry-wet-dry cycle. One explanation could be that not all the hydrogen bonds broken due to supercontraction form again when re-wetted and restretched. Network has lower inter-chain hydrogen bonds to act as a constraint to align the protein chains in the preferred orientation compared to the original dry silk. The authors have observed a similar pattern of structural hysteresis due to supercontraction in the in the MA silk of the spider Lactrodectus Hesperus (Black Widow). ${ }^{66}$

In a combined FTIR spectroscopy and dynamical mechanical properties measurement on the MA silk of Nephila edulis, in the dry and supercontracted states, Ene $e t a l$. have shown that when stretched from the supercontracted state, the dynamic elastic modulus does not return to its original value as the in dry native state. $^{20}$

It is noteworthy that Argiope aurantia MA silk which has a higher amount of the amino acid proline and a higher crystallinity than the Nephila clavipes MA silk, has a higher structural hysteresis due to supercontraction.

Comparing the behaviour of the silks from the two species Nephila clavipes and Argiope aurantia, we find that the overall trend in the structural change in the MA and MiA silks are 
similar through the process of supercontraction. MA silks in both Nephila clavipes and Argiope aurantia supercontract significantly more than their corresponding MiA silks. Orientation of the protein chains in the amorphous network does not return to its pre-supercontraction value in the MA silks of both Nephila clavipes and Argiope aurantia. While both primary sequence and the processing conditions are known to affect the fiber's structure and physical properties, we believe that the primary sequence plays a determining role in secondary structure of the protein chains which in turn determines the tertiary structure - crystalline and amorphous components. Amount of proline in the fiber has been suggested to affect mechanical properties in MA silks, ${ }^{7,59,60,62-65}$ and higher proline in the fiber, higher the shrinkage in water. However our results show that the crystalline/amorphous ratio in the fiber also seem to be an influencing factor on the ability of the fiber to regain its nanostructure after supercontraction and restretching. Whether proline is a factor in determining the ratio of crystalline/ amorphous components, has not been investigated so far. While it is important to understand fundamental molecular mechanisms governing the supercontraction process, from an applications perspective, knowledge that the MA silks do not regain its original structure completely after supercontraction has to be taken into account and compensated for in the biomimetic design for synthetic fibers. Also, MiA silks contract to a lesser extent in water, and can be used as an advantage while considering applications under wet conditions for example, as sutures for surgery.

\section{Summary of results and conclusion}

Structural changes through the process of supercontraction have been studied using WAXD on spider silks from MA and MiA glands from two different species Nephila clavipes and Argiope aurantia. Molecular chain orientation is an important structural parameter, which affects the structure and function of the silk fibers. Salient features in the results are summarized as:

(i) MA silks in both Nephila clavipes and Argiope aurantia supercontract significantly more than MiA silks.

(ii) MA silks are more resilient to the dry-wet-restretched dry cycles. Immersion in water induces an irreversible change in the MiA silks, leading them to break during wet-restretching from the contracted state.

(iii) Supercontraction leads to loss of orientation in both crystalline and amorphous components in all silk fibers studied. The loss of orientation in the oriented-amorphous component is higher than that in the $\beta$-sheet nano-crystallites.

(iv) Wet-restretching from supercontracted state does not result in the micro-structure of the silk returning to its original structure as in its dry-native state (as thought by previous structural and mechanical properties studies).

(v) In the MA silks, the $\beta$-sheet nano-crystallites regain their preferred orientation after supercontraction, while the oriented amorphous component does not regain its dry state value, resulting in a structural hysteresis caused by water. (vi) Percentage change in orientation in the amorphous component between the dry native state and wet-restretched state is greater in Argiope aurantia MA silk than Nephila clavipes MA silk. Interestingly, Argiope aurantia MA silk has higher proline content and also higher crystallinity.

Careful measurements and detailed data analysis have elucidated that the degree of orientation of the oriented amorphous component is not only an important structural parameter affecting structure and properties in the dry silk, but plays a significant role in determining the structure in the supercontracted and restretched states. We hope that these results will a test-bed for atomistic modelling studies to correlate sequence to structure and further help understand the role of orientation of protein chains in different conditions (wet, dry) in the spider dragline silk.

\section{Acknowledgements}

The Department of Defense (DOD) Air Force Office of Scientific Research (AFOSR) under award no. FA9550-14-1-0014 and the National Science Foundation (NSF), Division of Materials Research under award no. DMR-1264801 supported this work. S.S. would like to acknowledge support in part by the M. Hildred Blewett Fellowship of the American Physical Society, "http://www.aps.org". Use of the Advanced Photon Source was supported by the U.S. Department of Energy, Basic Energy Sciences, Office of Science, under contract no. DE-AC02-06CH11357 and DOE-EPSCoR under contract number DE-SC0004791. Use of the BioCARS Sector 14 was supported by the National Institutes of Health, National Center for Research Resources, under grant number RR007707. The authors would like to thank Robert Henning for his help during the X-ray experiments, T. Isdebski for help with the samples. We would like to thank Prof. Randy Lewis and Prof. Greg Holland for helpful discussions.

\section{References}

1 R. W. Work, Text. Res. J., 1977, 47, 650-662.

2 Y. Liu, Z. Shao and F. Vollrath, Nat. Mater., 2005, 4, 901-905.

3 G. V. Guinea, M. Elices, J. Perez-Rigueiro and G. Plaza, Polymer, 2003, 44, 5785-5788.

4 T. A. Blackledge, C. Boutry, S.-C. Wong, A. Baji, A. Dhinojwala, V. Sahni and I. Agnarsson, J. Exp. Biol., 2009, 212, 1981-1989.

5 M. Elices, J. Pérez-Rigueiro, G. Plaza and G. V. Guinea, J. Appl. Polym. Sci., 2004, 92, 3537-3541.

6 M. Elices, G. R. Plaza, J. Perez-Rigueiro and G. V. Guinea, J. Mech. Behav. Biomed. Mater., 2011, 4, 658.

7 C. Boutry and T. A. Blackledge, J. Exp. Biol., 2010, 213, 35053514.

8 R. V. Lewis, Chem. Rev., 2006, 106, 3762-3774.

9 F. Vollrath and D. P. Knight, Nature, 2001, 410, 541-548.

10 M. Heim, L. Romer and T. Scheibel, Chem. Soc. Rev., 2010, 39, 156-164.

11 L. Eisoldt, A. Smith and T. Scheibel, Mater. Today, 2011, 14, 80-86. 
12 M. J. Buehler, Nano Today, 2010, 5, 379-383.

13 Z. T. Yang, O. Liivak, A. Seidel, G. LaVerde, D. B. Zax and

L. W. Jelinski, J. Am. Chem. Soc., 2000, 122, 9019-9025.

14 R. W. Work, J. Exp. Biol., 1985, 118, 379-404.

15 J. M. Gosline, M. W. Denny and M. E. DeMont, Nature, 1984, 309, 551-552.

16 A. H. Simmons, C. A. Michal and L. W. Jelinski, Science, 1996, 271, 84-87.

17 Y. Termonia, Macromolecules, 1994, 27, 7378-7381.

18 Y. Termonia, Structural biological materials, Amsterdam, Pergamon, 2000, ch. 9-11.

19 D. T. Grubb and G. Ji, Int. J. Biol. Macromol., 1999, 24, 203210.

20 R. Ene, P. Papadopoulos and F. Kremer, Soft Matter, 2009, 5, 4568-4574.

21 R. V. Lewis, Acc. Chem. Res., 1992, 25, 392-398.

22 R. W. Work, Journal of Arachnology, 1981, 9, 299-308.

23 G. V. Guinea, M. Elices, J. Pérez-Rigueiro and G. R. Plaza, J. Exp. Biol., 2005, 208, 25-30.

24 D. Bittencourt, B. M. Souto and N. C. Verza, Comp. Biochem. Physiol., Part B: Biochem. Mol. Biol., 2007, 147, 597-606.

25 M. Elices, G. R. Plaza, M. A. Arnedo, J. Perez-Rigueiro, F. G. Torres and G. V. Guinea, Biomacromolecules, 2009, 10, 1904-1910.

26 C. Smith, J. Ritchie, F. I. Bell, I. J. McEwen and C. Viney, Journal of Arachnology, 2003, 31, 421-424.

27 K. N. Savage, P. A. Guerette and J. M. Gosline, Biomacromolecules, 2004, 5, 675-679.

28 G. V. Guinea, M. Elices, G. R. Plaza, G. B. Perea, R. Daza, C. Riekel, F. Agullo-ÀRueda, C. Hayashi, Y. Zhao and J. PerÀez-Rigueiro, Biomacromolecules, 2012, 13, 2087-2098.

29 A. D. Parkhe, S. K. Seeley, K. Gardner, L. Thompson and R. V. Lewis, J. Mol. Recognit., 1997, 10, 1-6.

30 G. P. Holland, J. E. Jenkins, M. S. Creager, R. V. Lewis and J. L. Yarger, Biomacromolecules, 2008, 9, 651-657.

31 D. T. Grubb and L. W. Jelinski, Macromolecules, 1997, 30, 2860-2867.

32 C. Riekel, C. Branden, C. Craig, C. Ferrero, F. Heidelbach and M. Muller, Int. J. Biol. Macromol., 1999, 24, 179-186.

33 A. Bram, C. I. Branden, C. Craig, I. Snigireva and C. Riekel, J. Appl. Crystallogr., 1997, 30, 390-392.

34 C. Riekel and M. Muller, Macromolecules, 1999, 32, 44644466.

35 S. Sampath, T. Isdebski, J. E. Jenkins, J. V. Ayon, R. W. Henning, J. Orgel, O. Antipoa and J. L. Yarger, Soft Matter, 2012, 8, 6713.

36 J. M. Gosline, M. W. Denny and M. E. DeMont, Nature, 1982, 309, 551.

37 F. I. Bell, I. J. McEwen and C. Viney, Nature, 2002, 416, 37.

38 A. Hammersley, FIT2D, http:/www.esrf.fr/computing/expg.

39 L. Eisoldt, A. Smith and T. Scheibel, Mater. Today, 2011, 14, 80-86.

40 B. Madsen, Z. Shao and F. Vollrath, Int. J. Biol. Macromol., 1999, 24, 301-306.

41 F. Vollrath, B. Madsen and Z. Shao, Proc. R. Soc. London, Ser. $B$, 2001, 268, 2339-2346.
42 R. S. Stein and G. L. Wilkes, in Structure and properties of oriented polymers, ed. I. M. Ward, Applied Science Publishers Ltd, London, 1975, pp. 70-84.

43 M. R-Lacroix and C. Pellerin, Macromolecules, 2013, 46, 5561-5569.

44 I. M. Ward, Structure and Properties of Oriented Polymers, Chapman \& Hall, London, 2nd edn, 1997, pp. 1-25.

45 H.-R. Tseng, L. Ying, B. B. Y. Hsu, L. A. Perez, C. J. Takacs, G. C. Bazan and A. Heeger, J. Nanosci. Lett., 2012, 12, 63536357.

46 K. Kurabayashi, Int. J. Thermophys., 2001, 22, 277-288; J. D. Schieber, D. C. Venerus and S. Gupta, Soft Matter, 2012, 8, 11781-11785.

47 M. P. W. Wilson, Polymer, 1974, 15, 277-282.

48 G. Wu, J. D. Jiang, P. A. Tucker and J. A. Cuculo, J. Polym. Sci., Part B: Polym. Phys., 1996, 34, 2035-2047.

49 A. L. Simal and A. R. J. Martin, Appl. Polym. Sci., 1998, 68, 441-452.

50 Z. Shao and F. Vollrath, Polymer, 1999, 40, 1799-1806.

51 M. Xu and R. V. Lewis, Proc. Natl. Acad. Sci. U. S. A., 1990, 87, 7120-7124.

52 M. Hinman and R. V. Lewis, J. Biol. Chem., 1992, 267, 1932019324.

53 C. Y. Hayashi, N. H. Shipley and R. V. Lewis, Int. J. Biol. Macromol., 1999, 24, 271-275.

54 J. E. Jenkins, M. S. Creager, R. V. Lewis, G. P. Holland and J. L. Yarger, Biomacromolecules, 2010, 11, 192-200.

55 X. Shi, J. L. Yarger and G. P. Holland, J. Magn. Reson., 2013, 226, 1-12.

56 T. A. Blackledge and C. Y. Hayashi, J. Exp. Biol., 2006, 209, 2452-2461.

57 S. L. Stauffer, S. L. Coguill and R. V. Lewis, Journal of Arachnology, 1994, 22, 5-11.

58 K. S. Katti, C. Gu and D. R. Katti, Natural Polymers: Composites, ed. M. J. John and S. Thomas, Royal Society of Chemistry, 2012, ch. 2, p. 26.

59 A. E. Brooks, H. B. Steinkraus, S. R. Nelson and R. V. Lewis, Biomacromolecules, 2005, 6, 3095-3099.

60 Y. Liu, A. Sponner, D. Porter and F. Vollrath, Biomacromolecules, 2008, 9, 116-121.

61 X. Shi, J. L. Yarger and G. P. Holland, Chem. Commun., 2014, 50, 4856-4859.

62 K. N. Savage and J. M. Gosline, J. Exp. Biol., 2008, 211, 19371947.

63 J. Guan, F. Vollrath and D. Porter, Biomacromolecules, 2011, 12, 4030-4035.

64 M. Marhabaie, T. C. Leeper and T. A. Blackledge, Biomacromolecules, 2014, 15, 20-29.

65 J. Pérez-Rigueiro, G. R. Plaza, F. G. Torres, A. Hijar, C. Hayashi, G. B. Perea, M. Elices and G. V. Guinea, Int. J. Biol. Macromol., 2010, 46, 555-557.

66 S. Sampath, J. E. Jenkins, E. Butler, J. Kim, R. W. Henning, G. P. Holland and J. L. Yarger, Biomacromolecules, 2013, 14, 3472 . 